\title{
Assessing Accuracy Factors in Deformable 2D/3D Medical Image Registration Using a Statistical Pelvis Model
}

\author{
Jianhua Yao \\ National Institute of Health \\ Bethesda, MD USA \\ jyao@cc.nih.gov
}

\author{
Russell Taylor \\ The Johns Hopkins University \\ Baltimore, MD USA
}

\begin{abstract}
:
Deformable $2 D-3 D$ medical image registration is an essential technique in Computer Integrated Surgery (CIS) to fuse $3 D$ pre-operative data with $2 D$ intra-operative data. Several factors may affect the accuracy of $2 D-3 D$ registration, including the number of $2 D$ views, the angle between views, the view angle relative to anatomical objects, the co-registration error between views, the image noise, and the image distortion. In this paper, we investigate and assess the relationship between these factors and the accuracy of $2 D-3 D$ registration. We proposed a deformable $2 D-3 D$ registration method based on a statistical model. We conducted experiments using a hemi-pelvis model and simulated $x$-ray images. Some discussions are provided on how to improve the accuracy of $2 D-3 D$ registration based on our assessment.
\end{abstract}

Keyword: deformable $2 D-3 D$ medical image registration, accuracy assessment, statistical model

\section{Introduction and Background}

Medical imaging data in Computer Integrated Surgery (CIS) can be categorized in three classes: 1) preoperative data; 2) intra-operative data; and 3) postoperative data. Registration between different forms of data is crucial for a wide variety of CIS applications. CT and MR images are frequently used in clinical diagnosis and surgical planning, but their use as interventional imaging modalities has been limited due to the space concern in the operating room. Common modalities for guiding surgical interventions are X-ray fluoroscopes. These images are acquired in real time, but only present $2 \mathrm{D}$ information. A number of important anatomical features cannot be visualized well in 2D images, such as relative 3D location and orientation of anatomical landmarks. One method to provide $3 \mathrm{D}$ information during the intervention is to register and fuse pre-operative 3D images/models with intra-operative images. Basically, one must find the $2 \mathrm{D}-3 \mathrm{D}$ projective transformation that maps a $3 \mathrm{D}$ object onto one or more $2 \mathrm{D}$ projective images of the same ob- ject. This problem is called the $2 D-3 D$ registration problem.

Many researchers have investigated 2D-3D medical image registration using different techniques and over different anatomical regions. Gueziec et al. [1] registered a pre-operative CT scan of a femur with fluoroscopy Xray images using surface based techniques. Lavallee et al. [2] proposed a KD tree and distance map method to register 2D X-ray images with 3D solid volumetric models. Feldmar et al. [3] presented a unified framework for 2D3D registration of free form curves and surfaces. Hamadeh et al. [4] extended Feldmar's method by combining X-ray image segmentation and 2D-3D registration. Weese et al. [5] presented an intensity-based method for 2D-3D registrations. LaRose et al. [6] investigated realtime iterative X-ray/CT registration techniques. Zollei et al. [7] proposed a mutual information based 2D-3D registration algorithm which establishes the proper alignment via a stochastic gradient ascent strategy. Fleute et al. [8] proposed a deformable 2D-3D registration technique based on a statistical surface model.

Most of the prior work has focused on rigid 2D-3D registration of single-subject images or models, i.e., the goal has been to determine a rigid transformation (translation and rotation) between a coordinate system associated with a set of projection images and another coordinate system associated with a $3 \mathrm{D}$ volumetric scan or anatomical model of the same patient. Anatomical changes over time or deformations caused by patient motion can reduce the accuracy of such methods. A more fundamental limitation is that they break down if a patient-specific scan or model is not available.

The work reported in this paper focuses on the problem of deformable 2D-3D registration of a set of X-ray projection images with generic anatomical models incorporating statistical information about anatomical variation within a patient population. Applications of deformable 2D-3D registration include creation of patient-specific 3D models from X-rays for the purpose of surgical planning, postoperative analysis, or intraoperative registration in the presence of (predictable) deformations. 
Accuracy is essential in 2D-3D registration in order to correctly transfer pre-operative knowledge into surgical procedures. Compared to 3D-3D registration between two $3 \mathrm{D}$ images, 2D-3D registration between a 3D image and a set of 2D images has less information available and more parameters to compute. There are several factors that may affect the accuracy of 2D-3D registration, including the number of $2 \mathrm{D}$ views, the angles between $2 \mathrm{D}$ views, the view angles relative to the anatomy, the co-registration between $2 \mathrm{D}$ views, the image noise, and the image distortion. Very few investigations have been conducted to assess how these factors affect the accuracy of 2D-3D registration. Assessment of these accuracy factors could provide valuable information to help researchers improve their surgical setup and protocol.

In our investigation, we first built a statistical pelvis model from a collection of training CT images, and generated simulated x-ray images from a CT data set. We then performed a deformable 2D-3D registration between the pelvis model and the DRRs. We controlled the parameters in DRR generation to manipulate the accuracy factors in 2D-3D registration, and compared the registration results with a ground truth result to reveal the relationship between the accuracy factors and the 2D-3D registration.

The remainder of this paper is organized as follows. Section 2 briefly introduces the statistical pelvis model and its construction from a set of training images. Section 3 describes our deformable 2D-3D registration scheme. Section 4 presents our experiments and the assessment of accuracy factors in 2D-3D registration. Finally, section 5 discusses the result of our investigation.

\section{Statistical pelvis model and its construc- tion}

We designed a unique model representation to characterize both the boundary surface and the density distribution of anatomical structures. The model is represented as a tetrahedral mesh equipped with embedded density functions for each tetrahedron. Prior information of both shape properties and density properties is also incorporated in the model.

We proposed an efficient and practical "tetrahedral mesh reconstruction from contours" method to build tetrahedral meshes for bone structures. The method produces tetrahedral meshes with high flexibility to accommodate any anatomical shape. The meshes align naturally with cortical bone boundaries, and minimize residual errors associated with the density representation. We assigned an analytical density function for every tetrahedron. Currently, the density functions are 2nd-degree Bernstein polynomials in barycentric coordinates of the tetrahedron.

We designed a training strategy to compute a statistical model from a collection of training models. A model aligning procedure is first performed to map all training models into a common mesh structure, and a Principal Component Analysis (PCA) method is applied to compute the variability of both shape properties and density properties of the anatomical structure. Using the PCA method, the model can be approximated by a set of deformation parameters $\left\{b_{i}\right\}$ as:

$$
Y=M(\bar{Y}, b)=\bar{Y}+P b
$$

Where $Y$ is a model instance, $M(\bar{Y}, b)$ is the instantiation operation, $\bar{Y}$ is the mean model representation, and $P$ is the eigenvector matrix characterizing the prior information. By changing the deformation parameters $\left\{b_{i}\right\}$, we can get a model instance, which is also a deformed version of the mean model.

We have built a statistical hemi-pelvis model from eight training images. Details of the statistical model and its reconstruction method can be found in our previous publications $[9,10]$.

\section{Deformable $2 \mathrm{D} / 3 \mathrm{D}$ registration scheme}

Given a set of 2D X-ray images and an anatomical model, a transformation of the model needs to be determined so that the projections of the model on the 2D Xray planes align with the X-ray images. The registration can be treated as determining a set of transformation and projection parameters $\left(t_{x}, t_{y}, t_{z}, r_{x}, r_{y}, r_{z}, s_{x}, s_{y}, s_{z},\left\{b_{i}\right\}, c_{x}\right.$, $\left.c_{y}, p_{x}, p_{y}, f\right)$. The first nine parameters define an affine transformation, including translation $\left(t_{x}, t_{y}, t_{z}\right)$, rotation angle $\left(r_{x}, r_{y}, r_{z}\right)$, and scale $\left(s_{x}, s_{y}, s_{z}\right)$. The deformation parameter set $\left\{b_{i}\right\}$ of the model also needs to be optimized for the patient specific structure. The last five parameters describe the perspective projection geometry. The coordinate $\left(c_{x}, c_{y}\right)$ is the image center; $\left(p_{x}, p_{y}\right)$ is the pixel size; and $f$ is the focal length of the camera. $\left(c_{x}, c_{y}, p_{x}, p_{y}, f\right)$ are intrinsic camera parameters which can be determined in a separate calibration stage before the registration [11].

The overall deformable 2D-3D registration scheme between a statistical model and a set of fluoroscopic Xray images is outlined as follows:

1: The intrinsic parameters $\left(c_{x}, c_{y}, p_{x}, p_{y}, f\right)$ of the fluoroscope device are calibrated before the registration procedure.

2: A set of fluoroscopic X-ray images $\left\{I_{f}\right\}$ are acquired and the inherent geometric distortion is corrected [12].

3: The X-ray image planes are then co-registered to determine their relative poses with respect to a fixed coordinate system.

4: Digitally reconstructed radiographs (DRRs) $\left\{I_{d}\right\}$ of the statistical model $M$ are generated on the co-registered $\mathrm{X}$-ray image planes, and a similarity measure $f$ between DRRs $\left\{I_{d}\right\}$ and fluoroscopic X-ray images $\left\{I_{f}\right\}$ is evaluated. 
5: A non-linear optimization algorithm is employed to maximize the similarity measure between DRRs and X-ray images and obtain optimal transformation parameters for model $M$. The transformation parameters are split into groups (translation, rotation, scale and deformation). Only one group of parameters is optimized in one iteration. To further improve the efficiency and robustness of the registration, the process is run in a multiple-resolution framework. This involves first searching for the solution in a coarser Xray image and a lower LOD model, and then refining the solution in a series of finer resolution images and models. The algorithm is also implemented in a multiple-step-size manner, in which it starts with a large step size and gradually reduces the step size as it gets closer to the optimal solution. This scheme leads to a faster algorithm, which is also less likely to fall into a false local minimum.

6: Repeat step 4 and step 5 until the difference of similarity measures between two iterations is below a small threshold value.

This scheme involves several key techniques. Among those, fluoroscopic image distortion correction and image co-registration were described in a previous paper [12]. "Mutual Information" [13] was used as the similarity measure.

\section{Assessment of accuracy factors}

This section discusses computational experiments to assess the ability of our proposed deformable 2D-3D method to adapt a statistical 3D density model to a set of $2 \mathrm{D}$ projection images and its robustness with respect to various accuracy factors.

Any stage in the 2D-3D registration scheme may affect its accuracy. The view configuration of the 2D images, such as the number of views, the angle between views, and the view angle relative to the anatomical structure, may influence the entire registration process. The errors in the image acquisition and processing will also degrade the accuracy of the registration. These errors include the co-registration error of $2 \mathrm{D}$ views, the image noise and the image distortion. We call the view configuration and the error sources in image acquisition and processing "the accuracy factors" of the 2D-3D registration. Our objective is to investigate whether and to what extent these factors affect the accuracy of 2D-3D registration. Since it is difficult to control the accuracy factor in real X-ray images, we use simulated X-rays (DRRs) in the experiments. By controlling and manipulating the parameters in DRR generation, we can simulate the changes of accuracy factors and therefore obtain the assessment of their impact on the accuracy of the 2D-3D registration. In order to better understand the impact of each factor, we

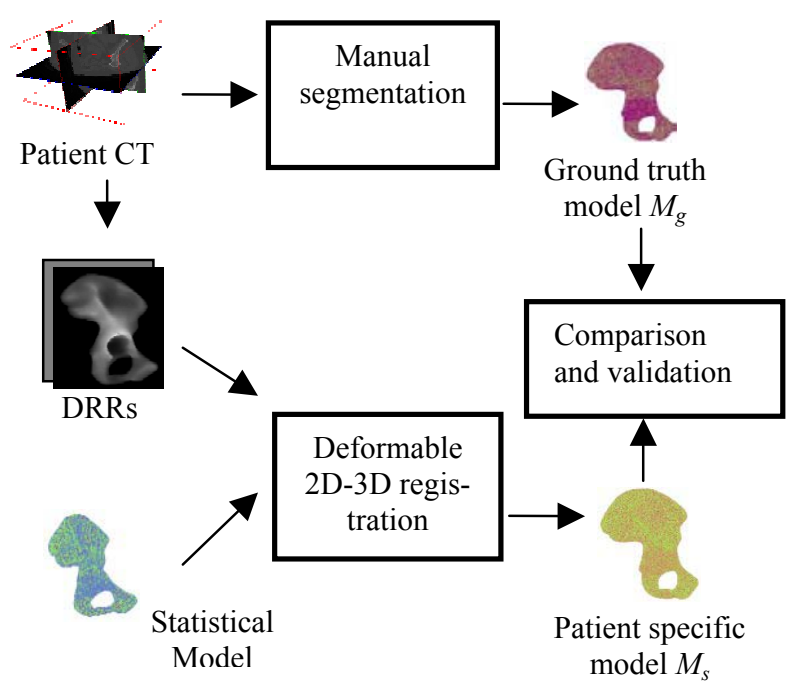

Figure 1. Experiment flow chart

only perturbed one factor at a time and kept other factors intact.

\subsection{Experiments and validation metrics}

In the experiment (Figure 1), first a patient CT image other than those in the training set is selected. Given a view configuration, a set of DRRs is generated from the CT image to simulate X-ray images. Then a deformable 2D-3D registration is conducted between the statistical model and the DRRs. The registered model $M_{s}$ is then a patient specific model. A manually segmented patient specific model $M_{g}$ from the patient CT image is used as the ground truth model. By comparing model $M_{s}$ with the ground truth model $M_{g}$, we can validate the accuracy of the $2 \mathrm{D}-3 \mathrm{D}$ registration.

In the experiment, we assumed that the intrinsic camera parameters (focal length, image center, and pixel size) are perfectly calibrated.

We used volume overlap percentage as our metric to compare two volumetric models. The volumetric model is scanned along $\mathrm{X}, \mathrm{Y}, \mathrm{Z}$ axes to produce a set of isotropic voxels within the model. The volume overlap is computed as the percentage of the number of overlapping voxels to the total number of voxels

$$
\text { Overlap }=\| V_{g} \text { I } V_{s}\|/\| V_{g} \| \times 100 \%
$$

here $V_{g}$ is the set of voxels in model $M_{g}, V_{s}$ is the set of voxels in model $M_{s}$, and $\|\cdot\|$ represents the size of a set.

\subsection{Number of 2D views}

We have investigated the relationship between the number of X-ray image views and the result of 2D-3D registration. It is expected that more views produce more 


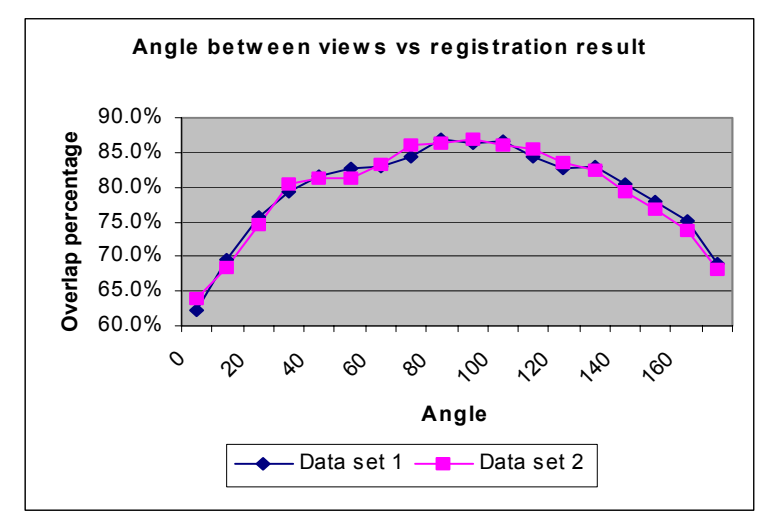

Figure 2. Angle between views vs. registration result

accurate results, but also slow down the procedure proportionally. If the number of views is large enough (e.g., greater than 50), the problem may be considered as a reconstruction problem rather than a registration problem. Table 5 shows the registration accuracy and the running time of using from one $2 \mathrm{D}$ view to six $2 \mathrm{D}$ views. The results demonstrate that as the number of views increases, the registration accuracy improves (the volume overlap increases), and the running time also increases proportionally. The improvement of volume overlap is significant from using one view to using two views, but is small after three views.

Table 1. Number of 2D views vs. registration results

\begin{tabular}{|c|c|c|c|c|c|c|}
\hline $\begin{array}{c}\text { Number of } \\
\text { views }\end{array}$ & 1 & 2 & 3 & 4 & 5 & 6 \\
\hline $\begin{array}{c}\text { Volume } \\
\text { overlap (\%) }\end{array}$ & 64.2 & 86.3 & 87.9 & 88.4 & 89.3 & 90.1 \\
\hline $\begin{array}{c}\text { Running } \\
\text { time (s) }\end{array}$ & 156 & 347 & 463 & 605 & 724 & 819 \\
\hline
\end{tabular}

\subsection{Angles between views}

The angles between X-ray views may affect the 2D$3 \mathrm{D}$ registration. We placed the $\mathrm{CT}$ data set of a pelvis in the center of two view planes. We then fixed one view plane and rotated the other view plane around the pelvis from $0^{\circ}$ to $180^{\circ}$ with $10^{\circ}$ increments starting from the angle of the fixed view plane, i.e., the angle between the two view planes changes from $0^{\circ}$ to $180^{\circ}$. At each angle, we generated two DRR images from two view planes and conducted a 2D-3D registration between the statistical pelvis model and the DRRs. Figure 2 plots the result of the registration versus the angle. The registration result is presented as the volume overlap percentage between the patient specific model produced by the $2 \mathrm{D}-3 \mathrm{D}$ registration and a ground truth model. The experiment is tested on two different CT data sets. As one would expect, the re-

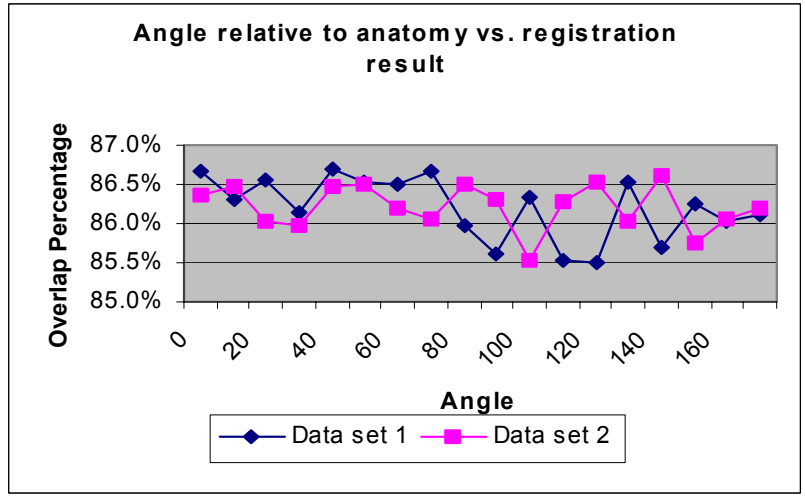

Figure 3. Angle relative to anatomical structure vs. registration result

sult shows that two X-ray views can produce best registration results when they are approximately orthogonal to each other. It produces the least accurate result at angle $0^{\circ}$ since it is equivalent to using just one view.

\subsection{View angles relative to the anatomical struc- ture}

We also investigated the impact of the view angles of $\mathrm{X}$-ray image planes relative to the anatomical structure on the registration results. We put a pelvis CT data set in the center of two view planes which orthogonal to each other. And we rotate the two view planes together around the pelvis from $0^{\circ}$ to $180^{\circ}$ with $10^{\circ}$ increments. $0^{\circ}$ is the angle where one view is the AP view and the other view is the lateral view. At each angle, we generate two DRR images and conduct the $2 \mathrm{D}-3 \mathrm{D}$ registration between the statistical model and the DRRs. Figure 3 plots the registration result versus the angle. The experiment is tested on two different CT data sets. The overlap percentages over different angles fluctuate around $86 \%$. The result shows that the view angle relative to the anatomical structure does not apparently affect the registration accuracy if we use two views orthogonal to each other. It is possible that the result might be different for different anatomy, and a simulation study is recommended in certifying this method for any particular clinical application.

\subsection{Image noise}

In the formation of an X-ray image, image noise is inevitable. The image noise may reduce the accuracy for the 2D-3D registration. To assess the effect of image noise on registration accuracy, we intentionally added 2D Gaussian white noise to the intensity values of DRR images. We then register the statistical model with the DRRs contaminated with noise. We tested different noise magnitudes (standard deviation of the Gaussian operator), and 


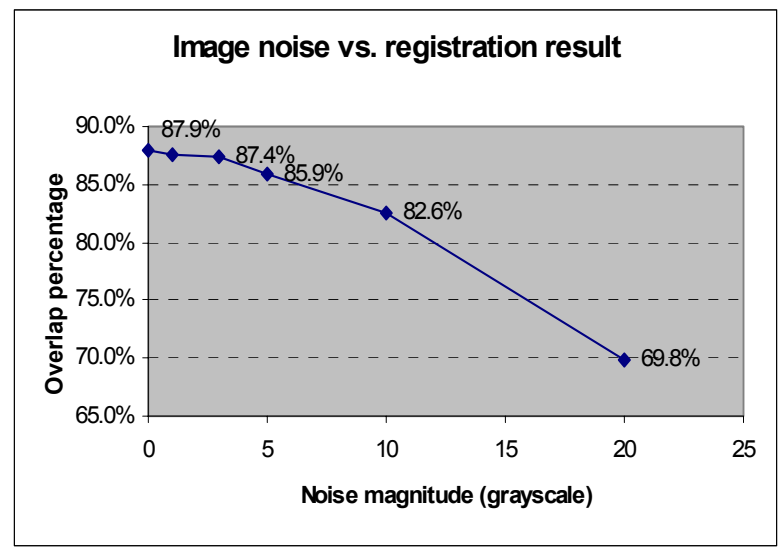

Figure 4. Image noise vs. registration result

plotted the relationship between the noise magnitude (in the units of grayscale) and the registration result (volume overlap percentage). We use three views in this experiment. The results in Figure 4 show that 2D-3D registration is insensitive to the noise when the noise magnitude is below 5 grayscale (about $2 \%$ of the maximum grayscale). However, the accuracy decreases dramatically for noise larger than 10 grayscale magnitude.

\subsection{Image distortion}

It is well known that fluoroscopic X-ray images have inherent spatial distortion due to the curvature of the image intensifier and the earth magnetic field effects. There are some existing algorithms to correct the spatial distortion in X-ray images, but small residual errors still exist after the correction [12]. We investigated the effect of spatial distortion of X-ray images on the 2D-3D registration by imposing spatial distortion to DRR images. We first get a normalized distortion map by applying a checkerboard plate based distortion correction algorithm, then given a distortion magnitude (in the unit of pixels), we multiply the distortion map with the magnitude and create a new distortion map; finally apply the new distortion map on the DRRs to generate distorted DRRs. We then register the statistical model with the distorted DRRs. Figure 5 plots the relationship between the magnitude of image distortion (in the unit of pixels) and the registration result (volume overlap percentage). The results show that the 2D-3D registration is sensitive to image distortion. Distortion of 10-pixelsize magnitude degrades the registration result dramatically.

\subsection{Co-registration error}

A set of X-ray images from different view planes need to be co-registered before they can be used in 2D-3D registration. The co-registration is usually conducted using magnetic or optical tracking devices, or calibration

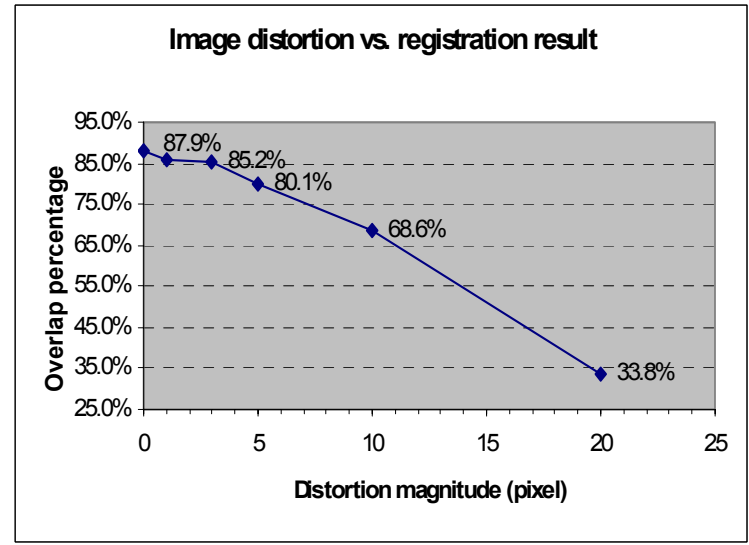

Figure 5. Image distortion vs. registration result

objects. There are residual errors in $2 \mathrm{D}$ image coregistration. We conducted experiments to assess the sensitivity of the 2D-3D registration to the residual error in 2D image co-registration. We used three views in our experiment, and added perturbations to the projection matrices of the view planes to simulate the errors in image co-registration. The rotation angle of the view plane relative to the pelvis was perturbed from $0^{\circ}$ to $20^{\circ}$. DRRs are generated using perturbed view matrices and registered with the statistical pelvis model. Figure 6 plots the relationship between the perturbation angles and the registration results. The results show that the $2 \mathrm{D}-3 \mathrm{D}$ registration is sensitive to the error in 2D image co-registration. A perturbation of $5^{\circ}$ can cause about $12 \%$ volume overlap decrease.

\section{Discussion}

We have proposed a deformable 2D-3D registration technique based on a statistical density model and validated our method through a series of experiments. We also assessed several accuracy factors that might affect the accuracy of 2D-3D registration.

We have identified several accuracy factors and assess their relationship with the accuracy of 2D-3D medical image registration. The results of this investigation can help researchers better understand the 2D-3D registration process and improve the setup and protocol to achieve more accurate 2D-3D registration. Six factors have been considered and assessed. They are the number of $2 \mathrm{D}$ views, the angle between views, the view angle relative to the anatomical structure, the X-ray image noise, the spatial distortion in fluoroscopic images, and the X-ray image co-registration error. In our investigation, we focused on the factors related to the $2 \mathrm{D}$ images. We haven't explored the factors related to the $3 \mathrm{D}$ images/models, such as the resolution and the variability of the model. But since the 3D images/models are acquired 


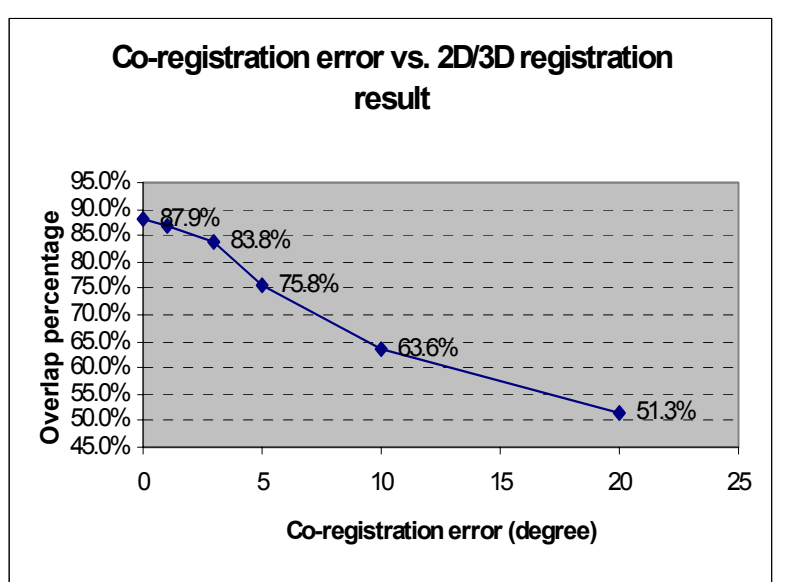

Figure 6. Co-registration error vs. registration result

or built before the intervention, their quality can be better controlled and guaranteed.

The more different $\mathrm{x}$-ray views are used, the more accurate the registration can be. But the number of available views is usually limited by the environment in the operating room, the surgical protocol and the patient position. From our results, three or four views are generally sufficient. As expected, there is a very big gain in going from one view to two, with diminishing returns after that. The experiments also show that if only two views are available, the best results are obtained when they are orthogonal. Since this is not always feasible, it is reassuring that the results are not greatly degraded so long as the viewing angle is between 75 and 105 degrees. The reason may be that the orthogonal views can compensate each other the content of the 3D object. The view angle relative to the anatomical structures has relative little impact on the registration. Since the angles to acquire X-ray images in the operating room should accommodate with the patient's position and other surgical instruments, sometimes there are not many view configurations to select. The effect of the presence of other anatomical structures or surgical instruments within the X-ray field of view, and their partial occlusion of the concerned anatomy, is yet to be investigated.

The insensitivity to small image noise in the 2D-3D registration may thank to the "mutual information" similarity measure used in the process. "Mutual information" utilizes the statistics of intensity distribution and smoothes out some of the noise. But excessive noise (more than $10 \%$ of the pixel intensity) will degrade the registration accuracy. Our study also shows that the 2D$3 \mathrm{D}$ registration procedure is relatively sensitive to the distortion in X-ray images. Fortunately, current distortion correction techniques can achieve about one-pixel accuracy, and new fluoroscope devices can generate X-ray images without spatial distortion [12]. The accuracy in 2D $\mathrm{X}$-ray co-registration is also a critical factor in 2D-3D registration. A 5-degree error in co-registration will introduce significant errors in following 2D-3D registration. The co-registration error can be minimized if a biplanar device is used. Overall, however, the results demonstrated a significant degradation of performance over a broad range of perturbation in co-registration and image distortion. It is highly possible to get acceptable 2D-3D registration if we have a set of accurately distortion corrected and calibrated X-ray images.

\section{References}

1. Gueziec, A., Assessing the registration of CT-scan data to intra-operative $X$-rays by fusing $X$-rays and preoperative information. Proceeding SPIE Medical Imaging'99, p. 3661-3688.

2. Lavallee, S., R. Szeliski, and L. Brunie, Anatomy-Based Registration of Three Dimensional Medical Images, Range Images, X-Ray Projections, and Three Dimensional Models Using Octree-Splines, in Computer-Integrated Surgery, R.H. Taylor, et al., Editors. 1995. p. 115-144.

3. Feldmar, J., N. Ayache, and F. Betting, 3D-2D projective registration of free-form curves and surfaces. 1994, INRIA, sophia antipolis: France.

4. Hamadeh, A., et al. Towards automatic registration between $C T$ and X-ray images: cooperation between $3 D / 2 D$ registration and $2 D$ edge detection. in 2 nd Annual International Symposium on MRCAS, 1995. Baltimore, MD USA.

5. Weese, J., et al. An Approach to 2D/3D Registration of a Vertebra in $2 D X$-ray Fluoroscopes with $3 D C T$ Images. in Computer Vision and Virtual Reality in Medicine II - Medical Robotics and Computer Assisted Surgery III, 1997. 1997. France.

6. LaRose, D., Iterative X-ray/CT registration using accelerated volume rendering, in Robotics Institute. 2001, Carnegie Mellon University.

7. Zollei, L. $2 D-3 D$ rigid registration of X-ray fluoroscopy and $C T$ images using mutual information and sparsely sampled histogram estimators. in IEEE CVPR, 2001. 2001.

8. Fleute, M. and S. Lavallee. Nonrigid 3-D/2-D Registration of Images Using Statistical Models. in MICCAI 1999. 1999. Cambridge, UK.

9. Yao, J., A statistical bone density atlas and deformable medical image registration, in Computer Science. 2001, the Johns Hopkins University.

10. Yao, J. and R. Taylor. Tetrahedral Mesh Modeling of Density Data for Anatomical Atlases and Intensity-Based Registration. in MICCAI 2000. 2000. Pittsburgh, PA, USA: Springer.

11. Tsai, R.Y., A Versatile Camera Calibration Technique for High-Accuracy $3 D$ Machine Vision Metrology Using Offthe-Shelf TV Cameras and Lenses. IEEE J. of Robotics and Automation, 1987. RA-3(4): p. 323-358.

12. Yao, J., et al., A C-arm fluoroscopy-guided progressive cut refinement strategy using a surgical robot. Computer Aided Surgery, 2000. 5(6): p. pp 373-390.

13. Wells, W., et al., Multi-modal volume registration by maximization of mutual information. Medical Image Analysis, 1996. 1: p. 35-51. 